\title{
INTEGRATION OF SEASONAL AUTOREGRESSIVE INTEGRATED MOVING AVERAGE AND BAYESIAN METHODS TO PREDICT PRODUCTION THROUGHPUT UNDER RANDOM VARIABLES
}

\author{
A. Azizi \\ Faculty of Manufacturing Engineering, \\ University Malaysia Pahang, 26600 Pekan, Pahang, MALAYSIA \\ E-mail: amirazizi@ump.edu.my \\ Phone: +6094245845; Fax: +609424 5888
}

\begin{abstract}
Analysing and modelling efforts on production throughput are getting more complex due to random variables in today's dynamic production systems. The objective of this study is to take multiple random variables of production into account when aiming for production throughput with higher accuracy of prediction. In the dynamic manufacturing environment, production lines have to cope with changes in set-up time, machinery breakdown, lead time of manufacturing, demand, and scrap. This study applied a Bayesian method to tackle the problem. Later, the prediction of production throughput under random variables is improved by the Seasonal Autoregressive Integrated Moving Average (SARIMA) method. The integrated Bayesian-SARIMA model consists of multiple random parameters with multiple random variables. A statistical index, R-squared, is used to measure the performance of the integrated model. A real case study on tile and ceramic production is considered. The Bayesian model is validated with respect to the convergence and efficiency of its outputs. The results of the analyses indicate that the Bayesian-SARIMA method produces a higher R-squared value, at $98.8 \%$, compared with previous studies on Bayesian methods where the value was $90.68 \%$ and the ARIMA method where it was $97.38 \%$. Consequently a robust approach in terms of the degree of prediction accuracy is proposed. This integrated method may be applied for the estimation of other production performance factors like lead time and cycle time in different types of dynamic manufacturing environment.
\end{abstract}

Keywords: Production throughput; breakdown; demand; lead time; scrap.

\section{INTRODUCTION}

Practically, the production rate at a workstation depends on random variables in the production line, which affect the final product throughput. The ability to handle random variables helps industrial engineers to accurately plan in order to meet customers' orders on time, thereby resulting in a competitive advantage for manufacturers. Industrial engineers have to match the production throughput with customers' orders by accurately predicting the throughput using a robust approach. However, current theories for handling and evaluating random variables and uncertainties under production throughput modelling are still under debate because these theories depend on the time factor $[1,2]$. Production throughput is considered an important parameter of production line performance [3-5]. Considering and handling the various production uncertainties on the shop floor are new challenges for academic research, and are known as complex 
optimization problems. In this study, the emphasis is on the production line random variables and uncertainties from the practical standpoint. This study focuses on the tile production industry. A more accurate model for estimating production throughput under the set-up time, scrap, break-time, demand, and lead time of manufacturing is derived using a Markov chain Monte Carlo (MCMC) algorithm for Bayesian-autoregressive integrated moving average (ARIMA) modelling. The overall operations for tile production are presented in Figure 1, which shows how raw materials including water and soil that is usually clay are mixed to provide slurry. Granule are made when the slurry is dried. When the granule is ready, the body of the tiles in the pressing stage is produced, namely, bisques. The bisques are moved to another stage called glazing and printing. The bisques are first sprayed with glaze. Glazes include frit, sand, kaolin, colouring agents, and chemical and mechanical resistance to prepare the bisque for firing. After spraying, the redundant glaze from the edges of the bisques is cleaned, and then they are transferred for printing. Printing is performed using different colours and lines (designs), which produce different types of tiles, along with gluing. Some types of tiles require two or three times of gluing and printing screens. When all this is done, the tiles are then transferred to a large kiln for firing. Finally, the tiles are ready for sorting and packing. Machines are subject to random failures, and set-up time is required to make changes for different product types in the real case study.

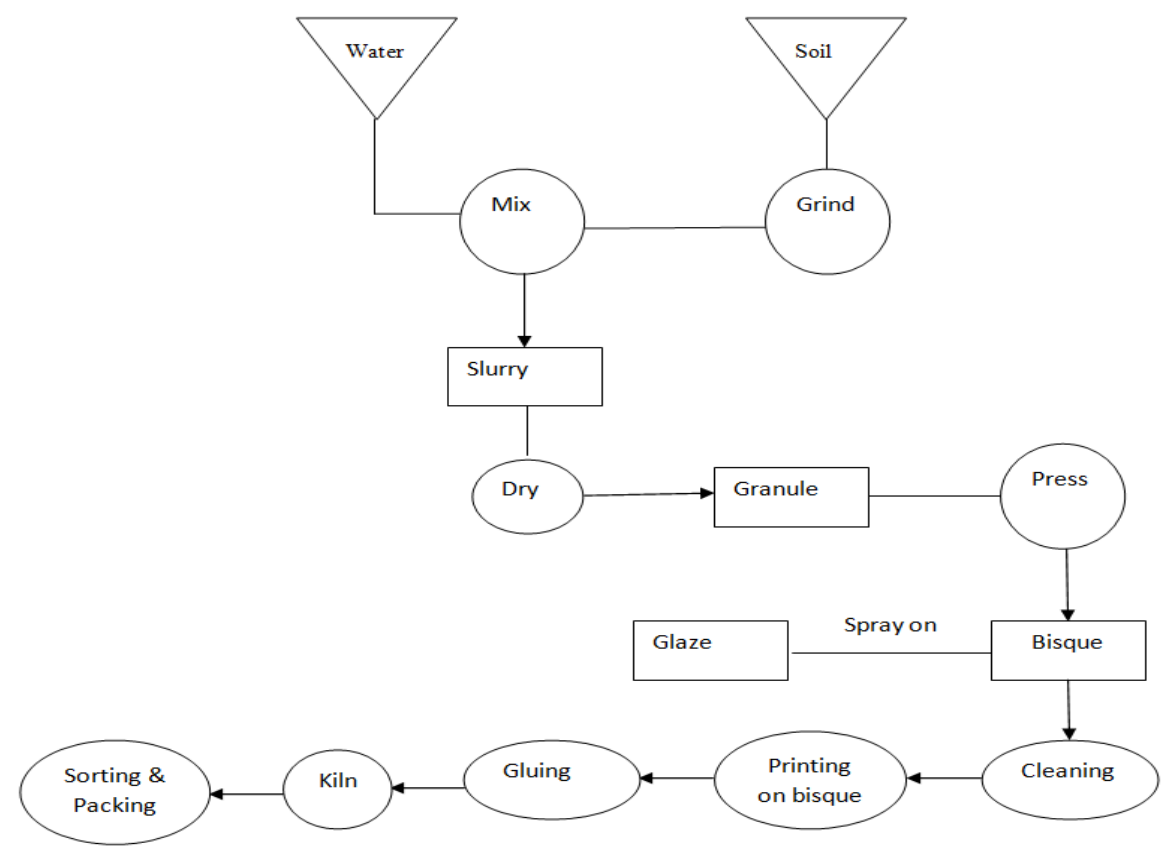

Figure 1. The flow of tile production.

\section{LITERATURE REVIEW}

Nowadays, the issue of how to handle production changes is becoming crucial. Studies show that processing time and breakdown time affect the production throughput $[3,6]$. Superior planning decisions are made by models that consider uncertainties and changes compared to models that do not [7]. Three uncertainties, namely, demand, manufacturing delay, and capacity scalability delay, are introduced by [8]. Demand changes, lead time variations, and uncertainty in resource breaks are surveyed in 
manufacturing environments and the results show that there are significant uncertain parameters [9]. An analytical algorithm was presented by Gilks, Richardson [10]. The authors predicted the production throughput under unbalanced workstations. A linear regression model was used for formulating strategy, environmental uncertainty, and performance measurement [11]. The Bayesian approach was explicitly used for external evidence in the design, monitoring, analysis, interpretation, and reporting of scientific investigations [12]. The most appropriate method in this context is MCMC, and is used in virtually all recently conducted Bayesian approaches [13]. The popular MCMC procedure is Gibbs sampling, which has also been widely used for sampling from the posterior distribution based on stochastic simulations for complex problems [14]. Gibbs sampling is used to solve complex statistical problems [15]. A few thousand iterations should be sufficient for moderate sized datasets involving standard statistical models [16].

\section{METHODOLOGY}

Bayesian inference is applied for this study. It uses a distribution-based approach where the prior probabilities are utilized to quantify uncertainty regarding the occurrences of events. The tile and ceramic industry is chosen because it is a real case study and it is under a dynamic production system and uncertainty. This industry comprises both manual and automated processes. The case study is located in the Alborz industrial city, Qazvin province, Iran. Data recorded over 78 weeks were found to be available for 20 highly requested types of tiles. More observational data were then continuously collected for a further 26 weeks for the same tile types. These data were collected for all six random variables: production throughput, breakdown time, lead time of manufacturing, demand, set-up time, and scrap. Once any breakdown time or changes occurred, they were recorded on a prepared form by the factory. Time was recorded using a clock watch/stopwatch. Then, at the end of the week, the occurrences were counted for each random variable, to be used for the following week's production plan. Thus, data recorded during 104 weeks were used as inputs for each random variable to estimate the production throughput. The ARIMA model was compiled with the Bayesian model, called a hybrid model. The best compilation of the hybrid model was considered based on generating the lowest Mean Absolute Percentage Error (MAPE). The improvements included changes to the values of the parameters $p$ and $q$ in ARIMA that were determined by the Autocorrelation Function (ACF) and Partial Autocorrelation Function (PACF). The algorithm procedure for the Bayesian-ARIMA approach is illustrated in Figure 2, which presents five random variables as inputs and one output which is the production throughput.

After collecting the observed data on both inputs and output, weakly informative priors [17] are suggested as the prior distribution of uncertainty to be considered for Bayesian inference, which is sampled by the Gibbs sampling method for a few thousand iterations as burn-in. The likelihood distribution of the observed data is calculated by the BUGS. The products of WIPs of uncertainty and the likelihood distribution of observed data with a few thousand iterations give the posterior distribution of uncertain parameters. Later, the model output is checked for validity by checking the convergence of two chains of sampling and efficiency of the Monte Carlo (MC) procedure by checking the error of MC, which should be less than 5\% of the standard deviation from the posterior mean estimation. If this is not valid or efficient, it may try for other distributions and a greater number of iterations. 


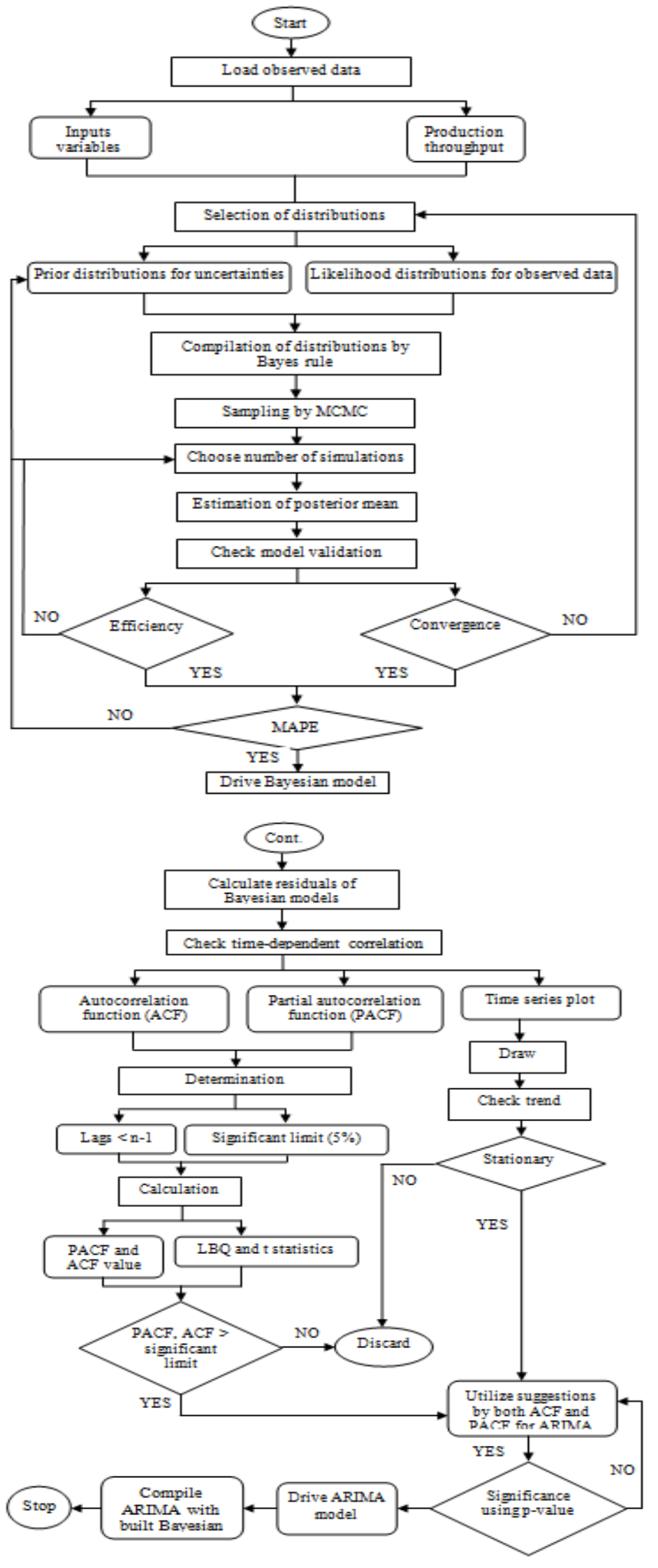

Figure 2. Bayesian-ARIMA approach algorithm. 
Subsequently, through the estimated posteriors, the production throughput is predicted. The difference between the predicted production throughput and actual production throughput is checked for time-dependent correlation using ACF and PACF in the ARIMA approach. The parameters of the ARIMA model are estimated with the significant time-dependent correlation of 5\%. Then the significance of the coefficients of ARIMA are checked by checking the t test and p-value. Finally, the estimated outputs of the ARIMA model are added to the predicted outputs of the Bayesian model.

\section{Number of Iterations for Sampling}

Four "burn-in" iterations: 1000, 5000, 8000, and 10,000, were examined. Iteration starts from 10,000 to 20,000 for drawing samples to approach convergence and reduce the Deviance Information Criterion (DIC) and MC error. Iteration started from 1000 and was increased until it reached convergence and the lower error of MC. The number of optimal iteration runs was determined by the higher level of convergence and the lower value of MC error and DIC. 10,000 iterations were carried out to generate initial values and 10,000 iterations were performed to maximize the posterior mean, starting from 10,001 to 20,000 .

\section{Bayesian Model Validation}

The model was validated through its convergence and the efficiency. Convergence was checked in three ways. The first checking was by visual inspection of the trace/history plots. The model's convergence was achieved when the two chains were overlapping. The convergence graphically presents how quickly the prior distributions of uncertainties approach the posterior distributions. The second checking was based on the autocorrelation test. The autocorrelation is defined between 0 and 1 or -1 . A slow convergence of two chains graphically shows the high autocorrelation within chains. It implies that two chains are mixed slowly because true distributions are defined. Thus, the mixed or convergence chain contains most of the information needed to estimate an accurate posterior distribution that indicates the validity of the model. The third checking used the Brooks Gelman Rubin (BGR) diagnostic. BGR numerically shows the convergence ratio, which should be near to 1 [18]. The idea is to generate multiple chains starting at over-dispersed initial values, and assess convergence by comparing within and between chain variability over the second half of those chains. According to Li, E. Blumenfeld [18], the BGR is calculated as shown in Eq. (1):

$$
B G R=\frac{W}{A}
$$

where $W$ is the width of the empirical credible interval of two chains based on all samples, and $A$ is the width average of empirical credible intervals across the two chains.

The efficiency of the model was checked by calculating the MC error. A lower value of MC error shows more accurate estimation of parameters. The MC error for each unknown parameter should be less than 5\% of the sample standard deviation [19], which indicates the model validation. The MC error for generating posterior parameters for each uncertainty is calculated by Eq. (2) according to [19]. 


$$
M C \text { error }=\frac{S D}{\sqrt{\text { Number of iterations }}}
$$

where $S D$ is the standard deviation.

Higher efficiency and lower MC error were achieved by adjusting the variances of prior distributions and number of iterations.

The following assumptions were considered for deriving the hybrid BayesianARIMA model.

- Normal distributions for priors were considered to enable comparison with the ANFIS model,

- Five random variables were considered based on the case study problem and availability of data for a long period of time (104 weeks) with reliable numbers of observations,

- Independent errors for random variables were assumed to be normally distributed.

\section{RESULTS AND DISCUSSION}

\section{Prior Probability Distribution of Uncertain Parameters}

WIPs are considered for prior distributions, because the advantage of WIPs is that the production management does not need to provide any prior opinions about the process. Different variances from 10 to 10,000 , which should be written as precisions of 0.1 to 0.0001 in BUGS, were tested for normal prior distributions based on the DIC. The best parameters were chosen according to the least DIC [15]. The prior distribution by the normal distribution is presented in Eq. (3) [20].

$$
P\left(\beta_{i}\right) \sim N\left(\mu, \delta^{2}\right)=\frac{1}{\delta \sqrt{2 \pi}} e^{-\frac{\left(\beta_{i}-\mu\right)^{2}}{2 \delta^{2}}}
$$

Table 1 presents the different variances of normal distributions and the calculated DIC respectively. Although set 1 resulted in lower DIC, as shown in Table 1, the other sets (different values given to the prior distributions) do not affect the DIC much. Thus, the prior distribution is correct because it has no substantial effect [21].

Table 1. Different parameters assigned as prior distributions.

\begin{tabular}{ccc}
\hline Sets & Variances & DIC \\
\hline 1 & $\beta_{0}$ and $\beta_{4}=10, \beta_{1}$ and $\beta_{3}=100, \beta_{2}$ and $\beta_{5}=1000$ & 1847 \\
2 & $\beta_{0}$ and $\beta_{4}=100, \beta_{1}$ and $\beta_{3}=1000, \beta_{2}$ and $\beta_{5}=10$ & 1848 \\
3 & $\beta_{\mathrm{i}}=100, \mathrm{i}=0, \ldots, 5$ & 1848 \\
4 & $\beta_{\mathrm{i}}=1000, \mathrm{i}=0, \ldots, 5$ & 1849 \\
5 & $\beta_{\mathrm{i}}=10000, \mathrm{i}=0, \ldots, 5$ & 1850 \\
\hline
\end{tabular}

The prior information on uncertainties with the normal distributions by means of zero and different variances ranging from 10 to 1000 is presented in Eq. (4-6). 


$$
\begin{aligned}
& \mathrm{P}\left(\beta_{0}\right)=\mathrm{P}\left(\beta_{4}\right) \sim \mathrm{N}(0,10) \\
& \mathrm{P}\left(\beta_{1}\right)=\mathrm{P}\left(\beta_{3}\right) \sim \mathrm{N}(0,100) \\
& \mathrm{P}\left(\beta_{2}\right)=\mathrm{P}\left(\beta_{5}\right) \sim \mathrm{N}(0,1000)
\end{aligned}
$$

The likelihood distributions of observations for uncertain variables are gained by integrating out the unknown parameter as shown in Eq. (7) [20]:

$$
P\left(u \mid \beta_{i}\right)=\frac{1}{\sigma \sqrt{2 \pi}} e^{-\frac{\left(u-\beta_{i}\right)^{2}}{2 \sigma^{2}}}
$$

The Bayes rule to postulate a prior on $\beta_{i}$ for the data observed for each uncertainty $(u)$ is presented as posterior distribution in Eq. (8) [20].

$$
P\left(\beta_{i} \mid u\right) \propto P\left(\beta_{i}\right) P\left(u \mid \beta_{i}\right) \propto \frac{1}{\delta \sqrt{2 \pi}} e^{-\frac{\left(\beta_{i}-\mu\right)^{2}}{2 \sigma^{2}}} \times \frac{1}{\delta \sqrt{2 \pi}} e^{-\frac{\left(u-\beta_{i}\right)^{2}}{2 \delta^{2}}}
$$

\section{Dynamic Trace Plot of Uncertain Parameters}

The convergence diagnostics were graphically checked through two chains of generated values. The convergence was achieved because both chains were overlapped with each other [18]. The dynamic trace plots of the stochastic parameters on 10,000 iterations are shown in Figure 3 with a 95\% credible interval. The history trace of 10,000 iterations of maximizing the posterior mean for all stochastic variables was checked for convergence too with a $95 \%$ credible interval. The convergence was approached because both chains look like a fat hairy caterpillar [22].
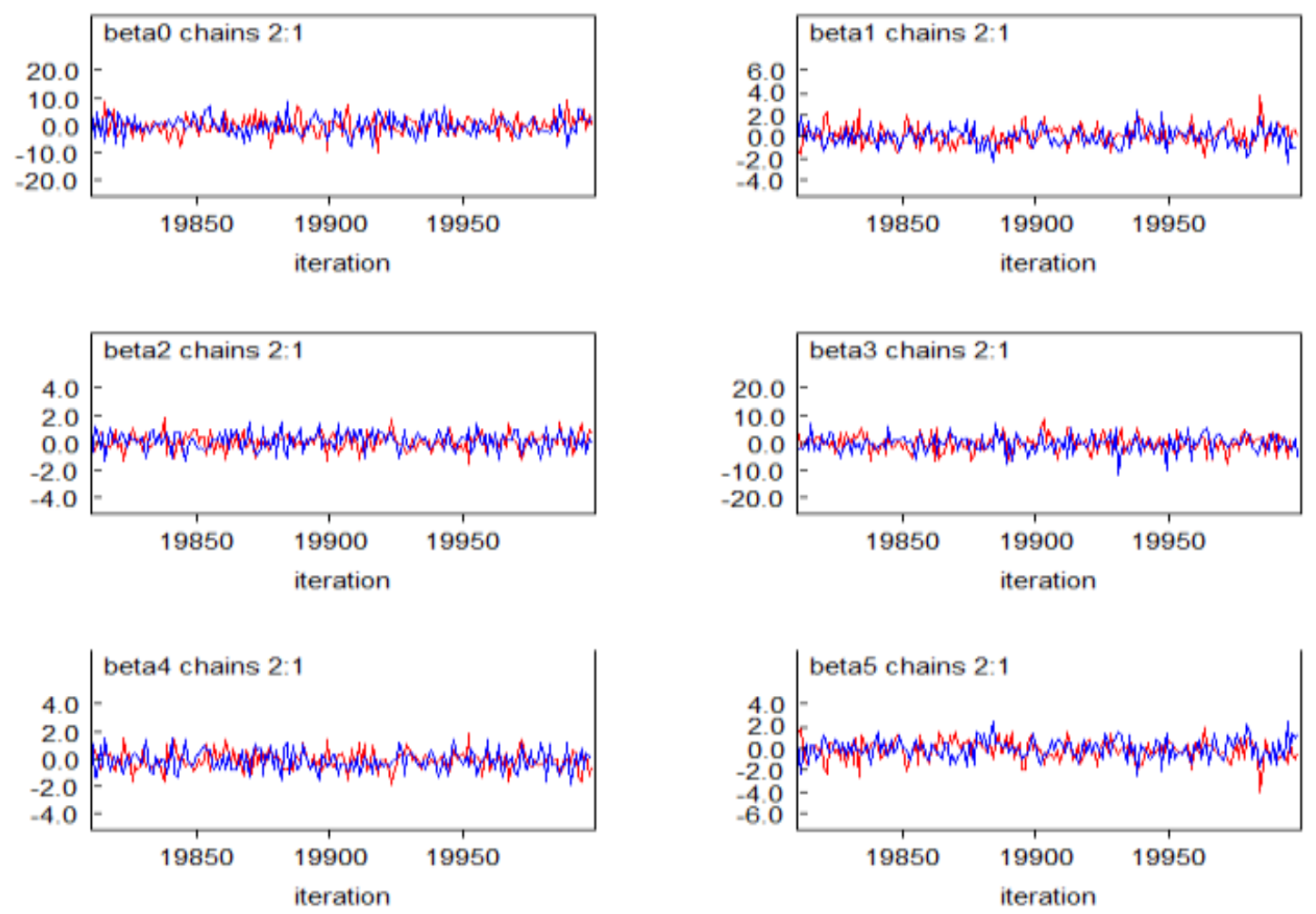

Figure 3. Dynamic trace plots of the stochastic parameters. 


\section{Autocorrelation Function of Uncertain Parameters}

The autocorrelation function plot for each uncertain parameter is shown in Figure 4 in two chains: blue and red. The plots indicate that the posterior distributions are gradually integrating, which implies high posterior correlations between parameters. The plots show that all uncertain parameters were properly integrated before 20 lags.
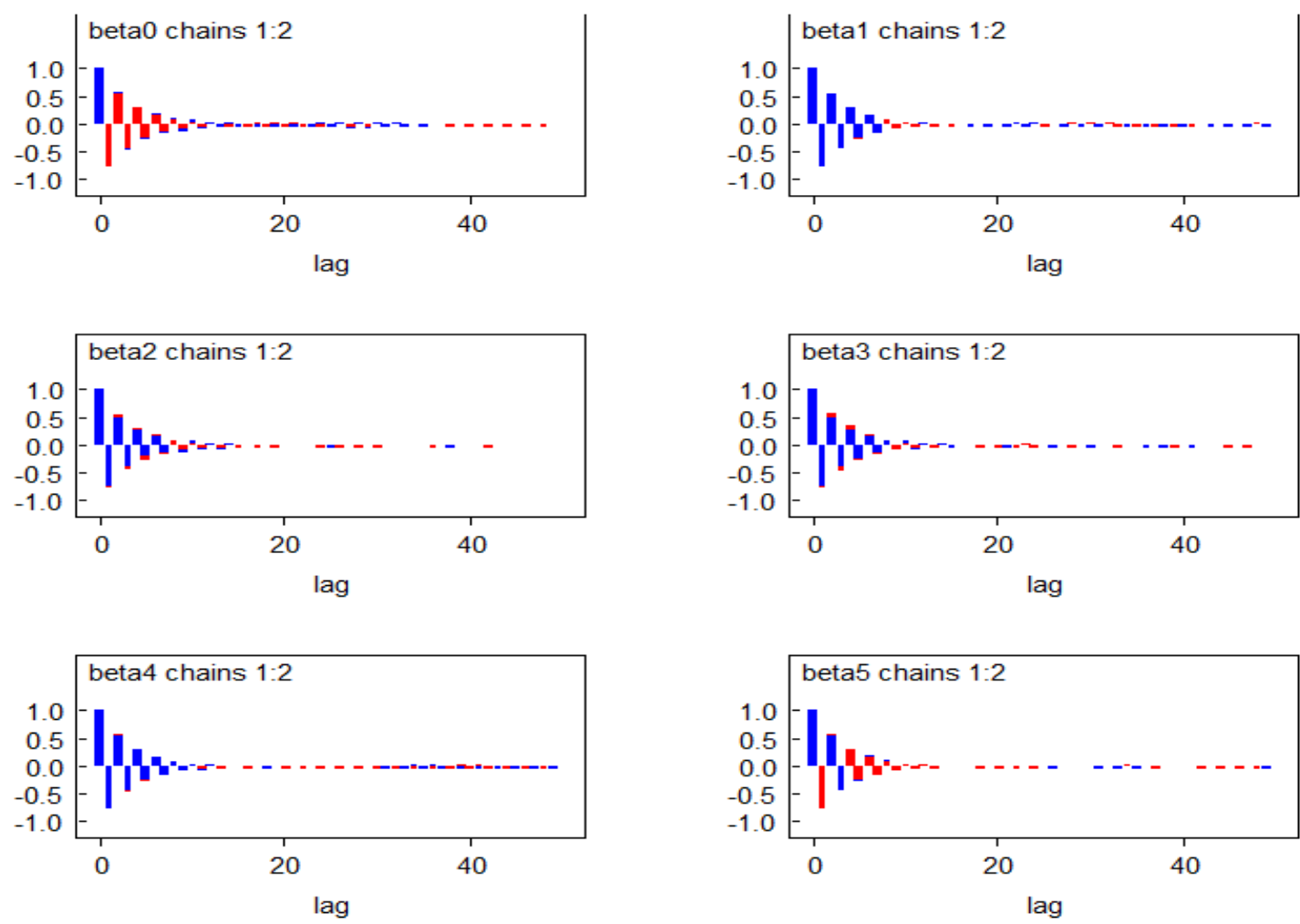

Figure 4. Autocorrelation function of the stochastic parameters.

\section{Brooks Gelman Rubin Statistics}

BGR statistics were calculated for all stochastic parameters. The calculated BGR was approaching 1 to prove that the number of iterations is enough and the model convergence was achieved [22]. Figure 5 shows that the chains of stochastic parameters approached convergence in most cases of iterations. The green line shows $W$ (normalized width of two chains) and the blue line exhibits $A$ (normalized mean within two chains), and the BGR is depicted by the red line. $W$ and $A$ were described under Eq. (1) as the BGR formula. The blue and green lines finally should be stabilized to tend to an approximately constant value [18]. When the iteration is increased, $W$ leads to $A$. Figure 5 shows that the green line is properly overlapped with the blue line especially after 12,000 iterations. This causes the BGR to become nearer to 1 . 

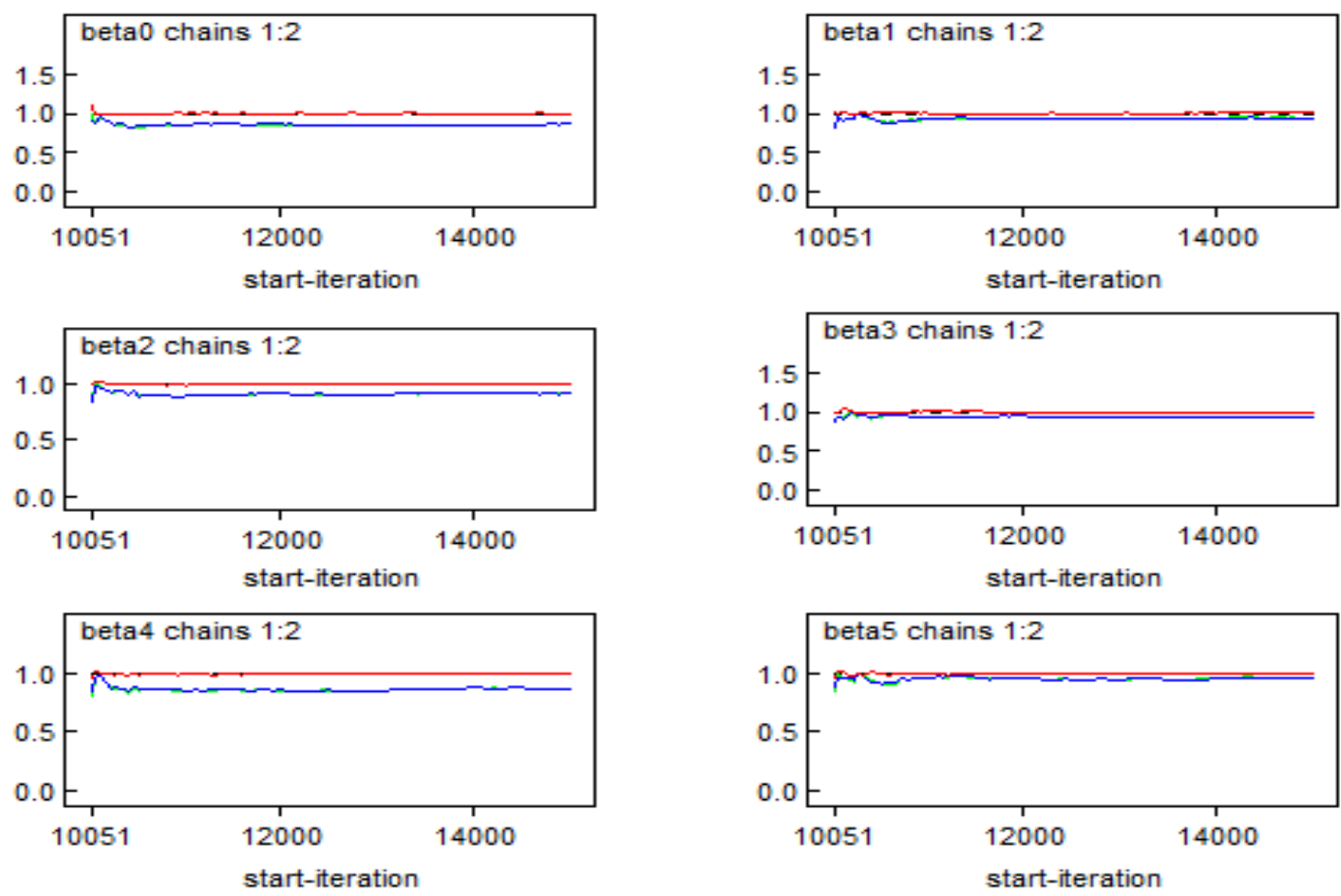

Figure 5. BGR statistics for uncertain parameters.

\section{Efficiency of the Bayesian Model}

Table 2 shows that the MC error for estimating the coefficient of intercept is about 0.0092 , and for the coefficients of breakdown time, demand, lead time, set-up time, scrap are $0.01033,0.00035,0.00132,0.00863$, and 0.00133 respectively. The Bayesian model shows high efficiency for the estimated coefficients of production uncertainties as the MC errors are less than $5 \%$ of the standard deviation of coefficients according to [19], as presented in Table 2.

Table 2. MC errors of uncertain parameters.

\begin{tabular}{cc}
\hline Coefficient & MC error \\
\hline$\beta_{0}$ & 0.0092 \\
$\beta_{1}$ & 0.01033 \\
$\beta_{2}$ & 0.00035 \\
$\beta_{3}$ & 0.00132 \\
$\beta_{4}$ & 0.00863 \\
$\beta_{5}$ & 0.00133 \\
\hline
\end{tabular}

\section{Estimates of Posterior Distributions of Uncertain Parameters}

The final set of posterior distributions estimations of production uncertainties using BUGS with $95 \%$ credible interval is summarised in Table 3 . The mean of the posterior distributions of $\beta_{i}$ is used for the Bayesian regression model because it minimizes the expected square loss according to Sheu and O'Curry [23]. Therefore, the Bayesian model developed is formulated in Eq. (9.): 


$$
\begin{gathered}
\widehat{P_{t, l}} \sim 0.00558-0.4704 B_{t, 1}+0.9526 D_{t}-0.1594 L_{t, 1}-0.01433 S e_{t, 1}- \\
0.1461 S_{t, 1}(t)+e_{t}
\end{gathered}
$$

where $e_{t} \sim N\left(0, \sigma^{2}\right)$

Table 3. Summaries of posterior distributions of uncertain parameters.

\begin{tabular}{cccccc}
\hline Coefficient & Mean & $S D$ & $5 \%$ of $S D$ & $2.5 \%$ & $97.5 \%$ \\
\hline$\beta_{0}$ & 0.00558 & 3.207 & 0.160 & -6.231 & 6.301 \\
$\beta_{1}$ & -0.4704 & 4.266 & 0.213 & -8.876 & 7.923 \\
$\beta_{2}$ & 0.9526 & 0.123 & 0.006 & 0.713 & 1.194 \\
$\beta_{3}$ & -0.1594 & 0.553 & 0.027 & -1.235 & 0.935 \\
$\beta_{4}$ & -0.01433 & 3.161 & 0.158 & -6.240 & 6.160 \\
$\beta_{5}$ & -0.1461 & 0.471 & 0.023 & -1.074 & 0.791 \\
\hline
\end{tabular}

The developed Bayesian model proposes a credible interval of changes for mean of uncertainties with a $95 \%$ credible interval in the following borders. $\beta_{1}$ has the widest prediction interval compared to other parameters, with the highest standard deviation of 4.266, as presented in Table 3. The ACF diagram is examined for the Bayesian residuals in Figure 6, which shows that there are significant autocorrelations in lags 1, 2, and 3 for Bayesian residuals with 5\% significance limits.

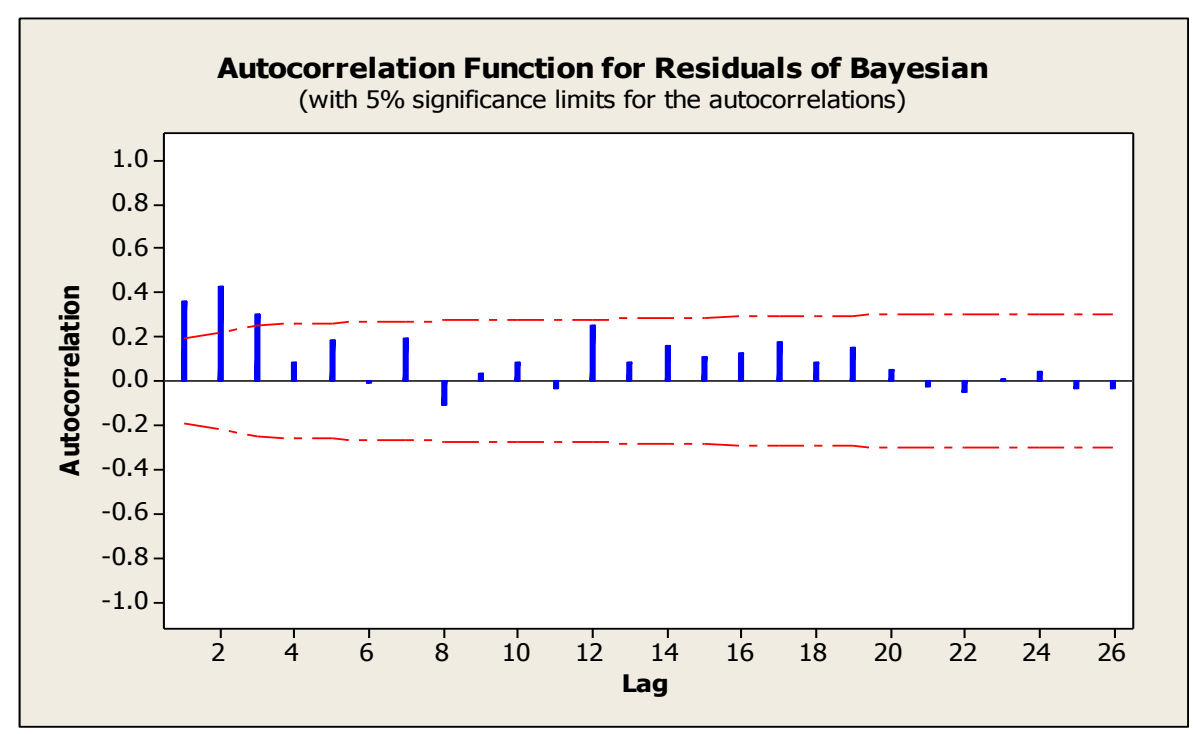

Figure 6. Autocorrelation function of Bayesian residuals.

The ACF values were calculated for the Bayesian residuals. This shows that the parameter numbers of the moving average for ARIMA modelling should be 1, 2 or 3 as the $t$ statistic values are greater than 1.96 based on a $95 \%$ confidence interval and their Ljung-Box-Q (LBQ) shows the smallest amount. The PACF for Bayesian residuals is also performed. The diagram of PACF of the Bayesian model is presented in Figure 7, which shows that there are significant partial autocorrelations in lags $1,2,7$, and 8 for Bayesian residuals with 5\% significance limits. 


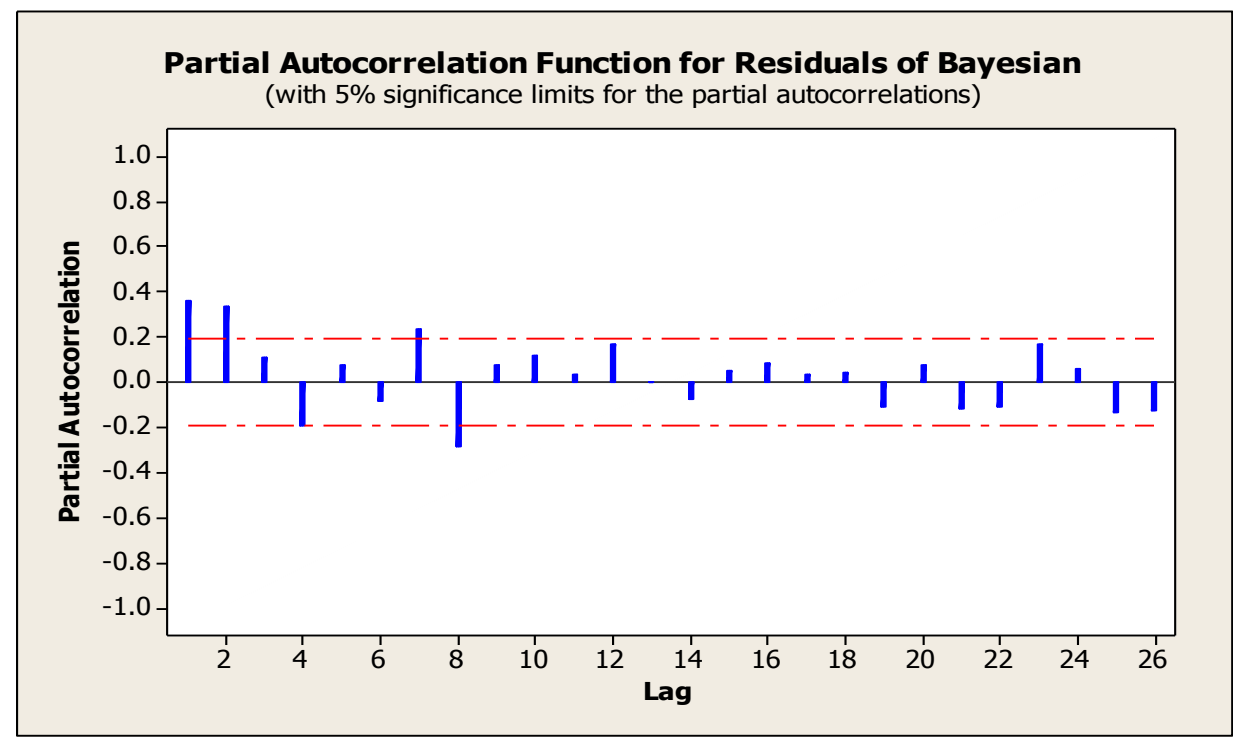

Figure 7. Partial autocorrelation function of Bayesian residuals.

The values of PACF for Bayesian residuals are calculated. This shows that the amounts of PACF for Bayesian residuals are significant with respect to 5\% significance limits in lags 1, 2, 7 and 8. Thus, according to the results of PACF as tabulated in Table 4, the candidates for the autoregressive parameter should be 1, 2, 7 or 8 because the $t$ statistic values are $3.67,3.44,2.38$, and -2.92 respectively, and are thus greater than the normal score of 1.96 or less than -1.96 based on a $95 \%$ confidence level. Therefore, the Bayesian residuals could be considered for ARIMA modelling in order to check if the utilization of the ARIMA approach could further increase the accuracy of the developed Bayesian model.

\section{SARIMA Model}

The modified ARIMA model was found in both the seasonal autoregressive and moving average. The final summaries of the coefficients of the SARIMA $(1,2)$ model are tabulated in Table 4.

Table 4. Final estimates of ARIMA parameters.

\begin{tabular}{|c|c|c|c|}
\hline Type & Coefficient & $t$ & $p$ \\
\hline SAR 12 & -0.9993 & -31.36 & $<0.0001$ \\
\hline SMA 12 & -1.6337 & -16.19 & $<0.0001$ \\
\hline SMA 24 & -0.7269 & -6.82 & $<0.0001$ \\
\hline Constant & 42.67 & 3.51 & 0.008 \\
\hline
\end{tabular}

where $S A R$ is seasonal autoregressive,

$S M A$ is seasonal moving average,

$t=t$ statistic.

$p=p$-value.

Table 4 shows that all the coefficients of the ARIMA model are optimum and significant, because their p-values are $<0.0001$ and for constant parameter it is 0.008 . 
Thus, the SARIMA model: SAR (1)12, SMA (2)12, and SMA (2)24 is formulated according to Zimmermann [24] in Eq. (10):

$$
\epsilon_{t} \sim 42.67-0.9993 \epsilon_{t-12}+a_{t}-1.6337 a_{t-12}-0.7269 a_{t-24}
$$

\section{Bayesian-ARIMA Model}

The hybrid Bayesian-ARIMA model is the combination of both the modified ARIMA model shown in Eq. (10.) and the developed Bayesian model presented in Eq. (10), as presented in Eq. (11.). The main benefit of this model is that it can consider time dependency and variations of uncertainties together because it accounts for the element of time compared to the Bayesian model individually.

$$
\begin{aligned}
\widehat{P_{t, l}} \sim 0.005581-0.4704 B_{t, 1}+0.9526 D_{t}-0.1594 L_{t, 1}-0.01433 S e_{t, 1} \\
-0.1461 S_{t, 1}+42.67-0.9993 \epsilon_{t-12}+a_{t}-1.6337 a_{t-12} \\
-0.7269 a_{t-24}+e_{t}
\end{aligned}
$$

Table 5 presents the accuracy of previous researches compared to this research. The accuracy of the developed Bayesian-ARIMA for this research is superior to the Bayesian and ARIMA in previous researches. In this study, more uncertain variables were taken into the model, which thus presents a stronger and more practical model with more observations, namely 104. More observations and more involved variables could produce a robust model with higher accuracy of estimation, indicated by $98.8 \%$.

Table 5. Comparison of previous approaches with the proposed approach.

\begin{tabular}{ccccccc}
\hline $\begin{array}{c}\text { Inputs } \\
\text { No. }\end{array}$ & $\begin{array}{c}\text { Outputs } \\
\text { No }\end{array}$ & observations & $\mathrm{R}^{2}$ & Approaches & Industry & References \\
\hline 1 & 1 & 17 & $90.68 \%$ & Bayesian & Lath & {$[23]$} \\
2 & 1 & 85 & $97.38 \%$ & ARMA & Automotive & (Popova, 2000) \\
5 & 1 & 104 & $98.8 \%$ & $\begin{array}{c}\text { Bayesian- } \\
\text { ARIMA }\end{array}$ & Tile & This research \\
\hline
\end{tabular}

\section{CONCLUSIONS}

This study found that the combination of the Bayesian inference and ARIMA approach on detecting production uncertainties and their impacts on the production throughput were as viable and accurate as the Bayesian and ARIMA approaches individually. The study modelled the propagation of uncertainties in a serial tile production line consisting of five random variables: demand, breakdown time, scrap, set-up time, and lead time, using a real case study on the tile industry in Iran. The hybrid model provides management with a clear picture of the variability inherent in the production processes. The proposed model is used to accurately predict the production throughput, and discover the mathematical relationship between the production uncertainties and throughput. The proposed hybrid model (Bayesian-ARIMA) demonstrated accuracy with an R-squared value of $98.8 \%$. Therefore, the Bayesian-ARIMA is recommended for production estimation under random variables and uncertain parameters of production. 


\section{ACKNOWLEGEMENTS}

The author would like to thanks to Universiti Malaysia Pahang for providing laboratory facilities and financial support.

\section{REFERENCES}

[1] Alden J. Estimating performance of two workstations in series with downtime and unequal speeds. General Motors Research \& Development Center, Report R\&D-9434, Warren, MI. 2002.

[2] Baker KR, Powell SG. A predictive model for the throughput of simple assembly systems. European Journal of Operational Research. 1995;81:336-45.

[3] 'Blumenfeld DE, Li J. An analytical formula for throughput of a production line with identical stations and random failures. Mathematical Problems in Engineering. 2005;2005:293-308.

[4] Brooks SP, Gelman A. Alternative methods for monitoring convergence of iterative simulations. 1998.

[5] Bolstad WM. Introduction to Bayesian statistics. New Jersey: John Wiley \& Sons; 2004.

[6] Dantzig GB. Planning under uncertainty. Annals of Operations Research. 1999;85:0-.

[7] Tan HB. Business and economic forecasting: techniques and applications. Serdang, Selangor Darul Ehsan: Penerbit Universiti Putra Malaysia; 2006.

[8] Congdon P. Bayesian Statistical Modelling. 2 ed. England: John Wiley \& Sons; 2007.

[9] Deif AM, ElMaraghy HA. Modelling and analysis of dynamic capacity complexity in multi-stage production. Production Planning \& Control. 2009;20:737-49.

[10] Gilks WR, Richardson S, Spiegelhalter D. Markov Chain Monte Carlo in practice. New York: Taylor \& Francis; 1995.

[11] Gelman A, Carlin JB, Stern HS, Dunson DB, Vehtari A, Rubin DB. Bayesian data analysis. Third Edition. Florida: Taylor \& Francis; 2013.

[12] Hoque Z. A contingency model of the association between strategy, environmental uncertainty and performance measurement: impact on organizational performance. International Business Review. 2004;13:485-502.

[13] Koop G, Steel MF, Osiewalski J. Posterior analysis of stochastic frontier models using Gibbs sampling. Computational Statistics. 1992;10:353-73.

[14] Koh SCL, Gunasekaran A. A knowledge management approach for managing uncertainty in manufacturing. Industrial Management \& Data Systems. 2006;106:439-59.

[15] Li L, Chang Q, Xiao G, Ambani S. Throughput bottleneck prediction of manufacturing systems using time series analysis. Journal of Manufacturing Science and Engineering. 2011;133:021015-.

[16] Li J, Blumenfeld DE, Alden JM. Comparisons of two-machine line models in throughput analysis. International Journal of Production Research. 2006;44:1375-98.

[17] Markel T, Brooker A, Hendricks T, Johnson V, Kelly K, Kramer B, et al. ADVISOR: a systems analysis tool for advanced vehicle modeling. Journal of Power Sources. 2002;110:255-66. 
[18] Li J, E. Blumenfeld D, Huang N, M. Alden J. Throughput analysis of production systems: recent advances and future topics. International Journal of Production Research. 2009;47:3823-51.

[19] Mula J, Poler R, García-Sabater JP, Lario FC. Models for production planning under uncertainty: A review. International Journal of Production Economics. 2006;103:271-85.

[20] Roberts GO. Markov chain concepts related to sampling algorithms. Markov chain Monte Carlo in practice: Springer; 1996. p. 45-57.

[21] Spiegelhalter DJ, Abrams KR, Myles JP. Bayesian approaches to clinical trials and health-care evaluation. New York: John Wiley; 2004.

[22] Spiegelhalter D, Thomas A, Best N, Gilks W. BUGS 0.5: Bayesian inference using Gibbs sampling manual (version ii). MRC Biostatistics Unit, Institute of Public Health, Cambridge, UK. 1996.

[23] Sheu C-f, O'Curry S. Simulation-based bayesian inference using BUGS. Behavior Research Methods, Instruments, \& Computers. 1998;30:232-7.

[24] Zimmermann HJ. Intelligent manufacturing management. In: Kahraman C, editor. Fuzzy Applications in Industrial Engineering: Springer Berlin Heidelberg; 2006. p. 383-400. 